\title{
Elastic stress concentration at radial crossholes in pressurized thick cylinders
}

\author{
T Comlekci*, D Mackenzie, R Hamilton, and J Wood \\ Department of Mechanical Engineering, University of Strathclyde, Glasgow, UK
}

The manuscript was received on 11 July 2006 and was accepted after revision for publication on 14 March 2007.

DOI: 10.1243/03093247JSA251

\begin{abstract}
Results of a parametric finite element analysis investigation of stress concentration at radial crossholes in pressurized cylinders are presented in numerical and graphical form. The analysis shows that the location of maximum stress does not generally occur at the junction between the bores, as is commonly supposed, but at some small distance up the crosshole from the junction. Maximum stress concentration factors (SCFs) are defined on the basis of the maximum principal stress, von Mises equivalent stress, and stress intensity. Three-dimensional plots of the SCF against the cylinder radius ratio $b / a$ and the crosshole-to-main-bore-radius ratio $c / a$ are presented. The SCFs were found to vary across the range of geometries considered with local minima identified within the parameter range in most cases. The results therefore allow designers to select optimum $b / a$ and $c / a$ ratios to minimize stress concentration in real problems.
\end{abstract}

Keywords: stress concentration factor, thick pressure vessels, crossholes

\section{INTRODUCTION}

Internally pressurized cylindrical components with radial holes through the cylinder wall, illustrated in Fig. 1, are used in a range of engineering applications, from small-scale components to large-scale plant. The Lamé solution for the elastic stress distribution in a pressurized cylinder is well known and given in standard strength of materials and elasticity texts, such as references [1] to [3]. However, when a crosshole is incorporated in the component, the stress distribution becomes much more complex, with a significant stress concentration occurring at the crosshole. Early analytical solutions for the stress concentration factor (SCF) at a radial crosshole combined the Lamé solution with elasticity solutions for stress concentration at a hole in a biaxially loaded flat plate [4-6]. In this type of analysis, the maximum SCF occurs at the intersection between the crosshole and the inner radius of the cylinder, on the plane of axial symmetry. Gerdeen [7] observed that the

\footnotetext{
* Corresponding author: Department of Mechanical Engineering, University of Strathclyde, 75 Montrose Street, James Weir Building, Glasgow, G1 1XJ, UK. email: Tugrul.Comlekci@strath.ac.uk
}

solutions proposed in references [4] to [6] lead to a common expression for (asymptotic) hoop stress SCF $K_{\theta}$ for small-diameter circular radial crossholes or sideholes (a radial penetration through one side of the cylinder only) given by

$$
K_{\theta}=\frac{\sigma_{\theta \max }}{\sigma_{\theta a}}=\frac{4(b / a)^{2}+1}{(b / a)^{2}+1}
$$

where $\sigma_{\theta \max }$ is the maximum hoop stress at the crosshole intersection, $\sigma_{\theta a}$ is the hoop stress at the inner surface of the main cylinder remote from the crosshole (from Lamé's equations), and $b$ and $a$ are the outer and inner radii respectively of the main cylinder. Gerdeen [7] extended this type of analysis to large sideholes and derived a solution applicable to a wide range of configurations. SCF curves for closed-end cylinders with sideholes obtained by Gerdeen are presented in Fig. 2. The form of the curves indicates that the magnitude of SCF decreases with increasing crosshole radius. Gerdeen suggested that extrapolation of these results indicates that the minimum theoretical SCF will be obtained for a cylinder with a sidehole ratio of $c / a=1$, i.e. the sidehole radius is equal to the radius of the cylinder bore. 


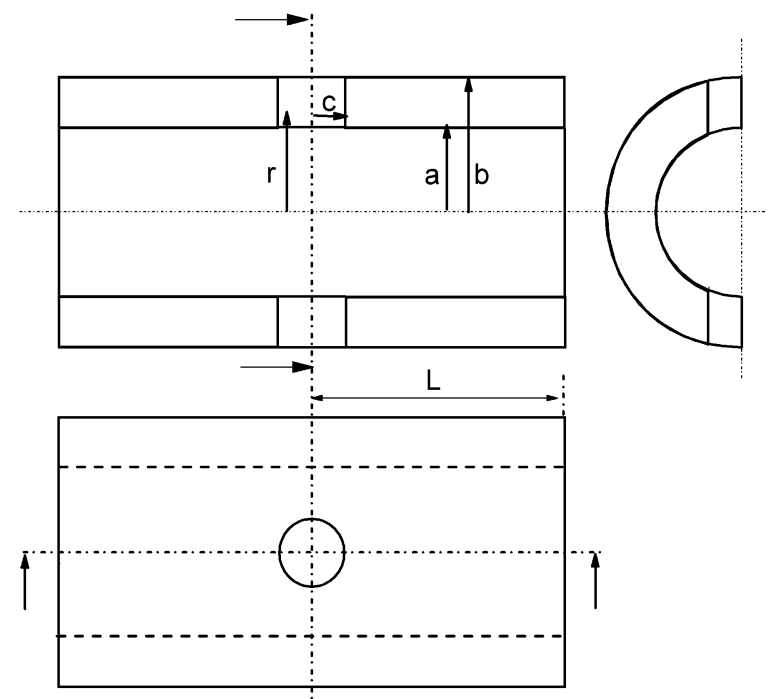

Fig. 1 Sectioned view of a thick cylinder with a radial crosshole

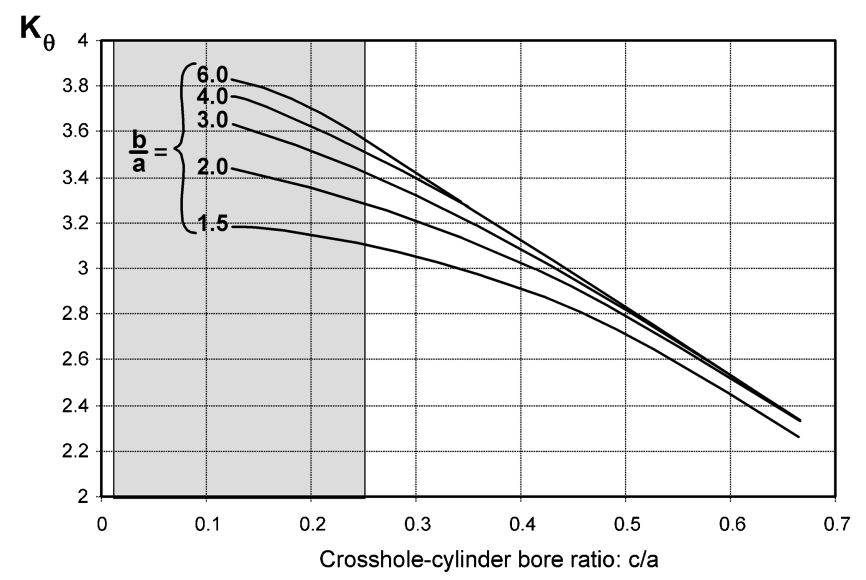

Fig. 2 Gerdeen's SCF curves for a radial crosshole in a pressurized cylinder (adapted from reference [7]), showing the $c / a$ range considered in the present study indicated as a grey region

Since Gerdeen's solution was published, the stress concentration at crossholes has been investigated by several workers using the finite element method. The effect of design features such as chamfers and blends at the junction between the crosshole and main bore on the SCF in particular have been studied in some detail [8-10]. Experimental strain gauge investigations of the SCF by Gerdeen and Smith [11] suggested that including a blend feature significantly reduced the SCF. However, fatigue test results [9] showed that vessels with a chamfer or blend have a marginally reduced fatigue life, indicating higher peak stress than found in a crosshole with a sharp intersection. The finite element analysis (FEA) results presented in references [9] and [10] show that incorporating a blend or chamfer reduces the stress concentration at the main bore (where the strain gauges were located in reference [11]) but a higher peak stress occurs at the junction between the blend and crosshole, leading to the reduced fatigue life observed in reference [8].

Although there has been a considerable amount of research into specific aspects of crosshole design, such as blending features, the SCF information available to designers for cylinders with sharp intersection crossholes is limited. Comparing the analytical equation (1) with FEA results from references [9] and [10] shows that this is really only accurate for very small crossholes. Gerdeen's solution curves from Fig. 2 were shown to be in good agreement with experimental analysis [11] but these experiments did not necessarily measure the actual maximum stress in the component, as discussed above. The object of this paper is to provide SCF data for a range of crosshole configurations representative of common applications by linear elastic three-dimensional FEA. Three types of SCF are considered for different design applications. Maximum principal stress SCFs are presented for design problems involving fracture mechanics or fatigue calculations. The von Mises yield criterion equivalent stress and the Tresca yield criterion stress intensity (SI) are presented for yield and fatigue calculations.

\section{CONFIGURATION AND ANALYSIS}

The investigation considers a thick cylinder, of inner radius $a$, outer radius $b$, and length $2 L$ with a central crosshole radius $c$, as shown in Fig. 1 . The radial through-thickness position is denoted $r: a \leqslant r \leqslant b$. The geometry parameters varied in the parametric investigation were the cylinder radius ratio $b / a$ and crosshole-to-bore-radius ratio $c / a$. The main cylinder internal radius was fixed at $a=100 \mathrm{~mm}$ in all analyses.

Seven moderate to thick cylinder radius ratios were considered: $b / a=1.4,1.5,1.6,1.75,2.0,2.25$, and 2.5. The crossholes investigated were relatively small, with the following crosshole-to-bore-radius ratios: $c / a=0.01,0.02,0.05,0.0750 .10,0.015,0.20$, and 0.25 . The range of crosshole sizes is smaller than that reported by Gerdeen [7], shown as the grey region in Fig. 2. These sizes are representative of a variety of practical engineering applications. Larger holes are likely to incorporate local reinforcement around the hole which may influence the stress distribution and for generality it was decided not to consider these in this paper. 


\subsection{Finite element model}

FEA was performed using the ANSYS program [12]. The configuration was modelled using threedimensional solid 20-node isoparametric brick elements, ANSYS Solid95. One eighth of the configuration was modelled with appropriate symmetry boundary conditions applied. A convergence study was conducted to establish the appropriate mesh density for the parametric study. Particular consideration was given to the number of elements through the cylinder thickness and the relative density of the mesh at the junction between the crosshole and main bore. A typical mesh, for $b / a=2$ and $c / a=0.2$, is shown in Fig. 3. This model has 24 elements through the thickness, with local mesh refinement in the junction region.

Internal pressure is applied to both the main bore and the crosshole. A plane-sections-remain-plane constraint was applied to the end of the cylinder remote from the crosshole and a uniform axial thrust corresponding to closed-end conditions applied. These boundary conditions are typical of those applied in earlier analytical studies [4-7]. However, the present study also assumes the crosshole to be
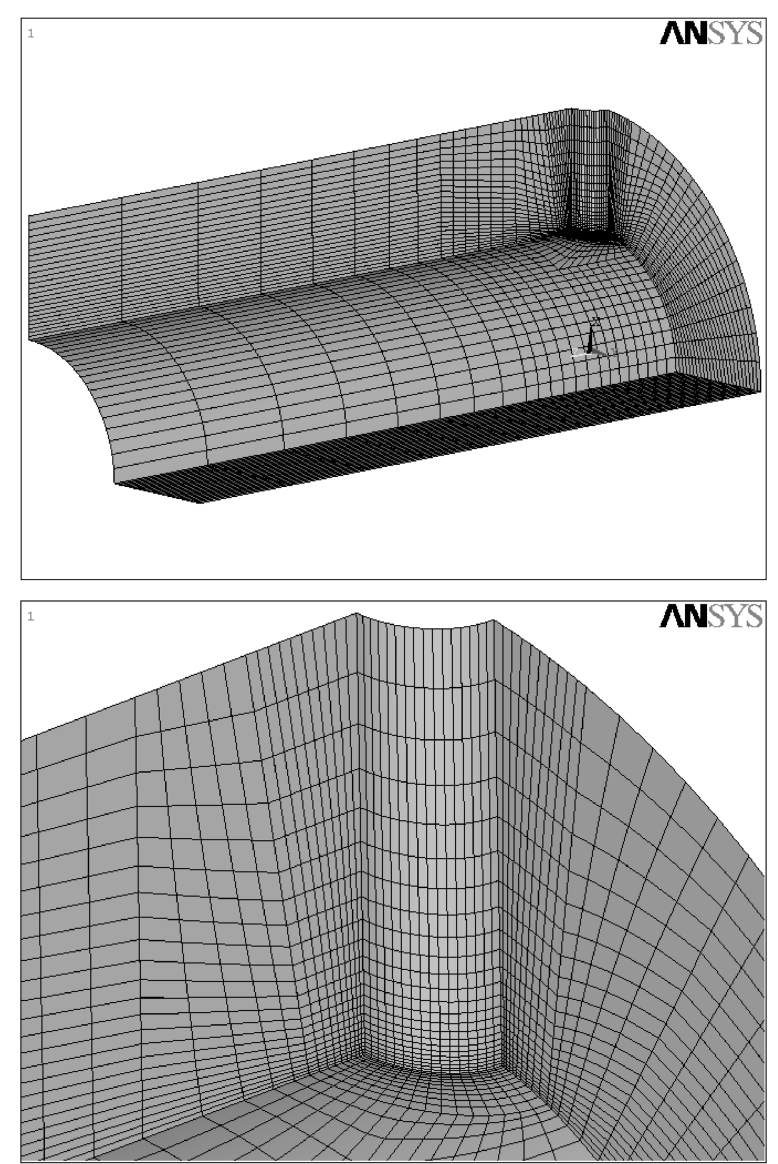

Fig. 3 Finite element mesh: $b / a=2 ; c / a=0.2$ closed at the outer surface. The corresponding radial thrust is applied to nodes around the crosshole at the outer surface. (This boundary condition does not have a major effect on the calculated SCF values for crossholes of the relative size considered.) A linear elastic material model was assumed, with Poisson's ratio $v=0.3$.

A significant number of the configurations considered in the ANSYS parametric investigation were also analysed using the Mechanica system [13]. This system uses adaptive- $P$ technology to achieve user-specified convergence result quantities. Convergence levels were defined at less than 1 per cent on displacement, strain energy, and an r.m.s. stress measure.

\section{RESULTS}

\subsection{Location of the maximum stress}

The parametric investigation showed that the maximum stress did not generally occur at the junction between the crosshole and main bore, as is implicitly assumed in analytical solutions. This is due to inclusion of $v$ in the analysis; when $v=0$, the SCF does occur at the junction. A similar effect where free surfaces give rise to a stress boundary layer has previously been identified in semianalytical solutions for SCF at holes in thick flat plates [14-16] but was not considered in closed-form crosshole solutions. It is also found that the strain results show similar trends to stress results where the maximum strain at a small crosshole in a pressurized cylinder did not generally occur at the junction between the crosshole and the main bore. This effect is illustrated for a specific configuration with $b / a=2$ and $c / a=0.2$ under unit internal pressure. A contour plot of first principal stress $\sigma_{1}$ in the vicinity of the crosshole is shown in Fig. 4. The highest stress contour band is seen to be located on the surface of the crosshole in the vicinity of the junction with the main bore; however, the contour plot does not show the location of maximum stress, $\sigma_{1 \max }=4.86 \mathrm{MPa}$, clearly. It is actually located on the crosshole surface at the plane of symmetry a short distance up from the intersection.

The distribution of $\sigma_{1}$ along a straight line from corners A to B in Fig. 4 is shown for a cylinder with the radius ratio $b / a=2$ for four crosshole-to-mainbore ratios $c / a=0.02,0.05,0.1$, and 0.2 in Fig. 5. The $c / a=0.20$ curve corresponds to the contour plot of Fig. 4 but shows the stress distribution more precisely. At the junction with the main bore $(r=100 \mathrm{~mm})$, 


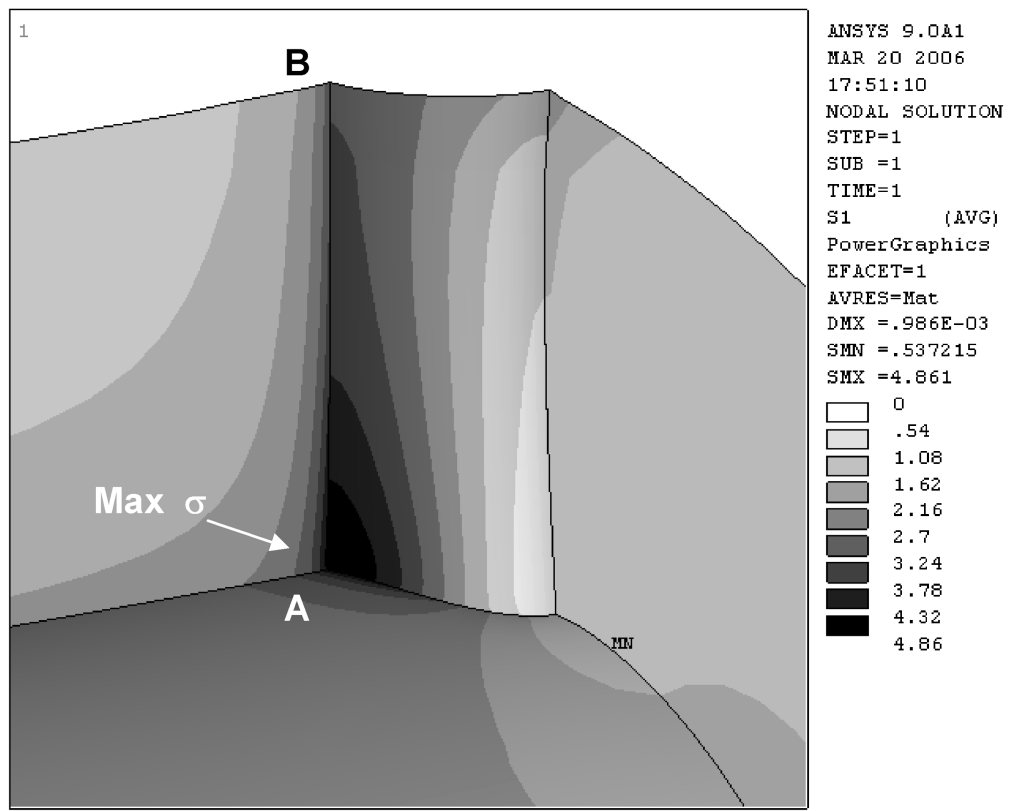

Fig. 4 Principal stress $\sigma_{1}$ contour plot for a cylinder with $a=100 \mathrm{~mm}, b=200 \mathrm{~mm}$, and $c=20 \mathrm{~mm}$ $(c / a=0.20)$ under unit internal pressure

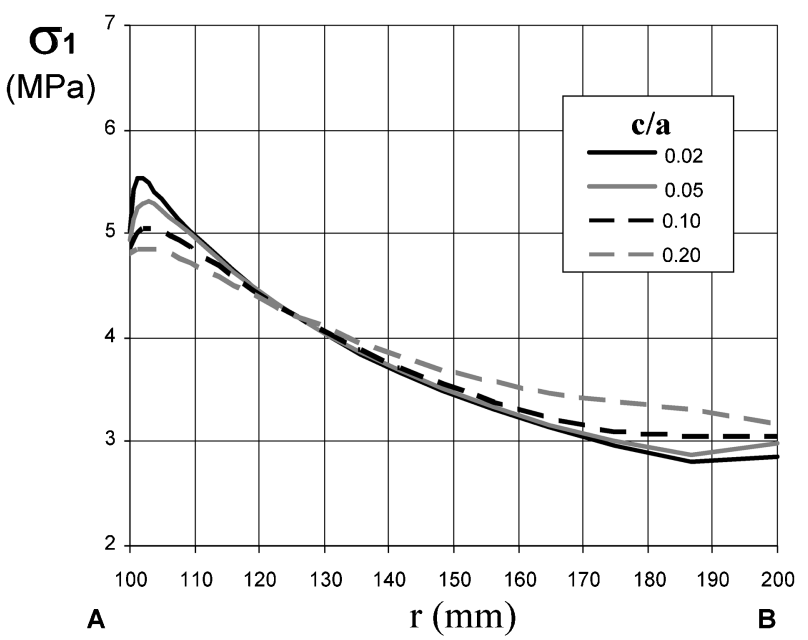

Fig. 5 Maximum principal stress $\sigma_{1}$ distribution on the crosshole surface on the axial symmetry plane from the inner radius $a=100 \mathrm{~mm}$ to the outer radius $b=200 \mathrm{~mm}$

$\sigma_{1}=4.82 \mathrm{MPa}$. This increases with $r$ to a peak value of $4.86 \mathrm{MPa}$ at $r=102 \mathrm{~mm}$ and then decreases to $3.16 \mathrm{MPa}$ at the outer surface $(r=200 \mathrm{~mm})$. The other three curves in Fig. 5 show that the location and magnitude of maximum stress concentration depends on the relative size of the crosshole. The smallest crosshole shown, $c / a=0.02$, gives the largest maximum stress of $\sigma_{1}=5.53 \mathrm{MPa}$ at $r=102 \mathrm{~mm}$. The corresponding stress at the intersection $(r=100 \mathrm{~mm})$ is significantly smaller: $\sigma_{1}=5.03 \mathrm{MPa}$. As the crosshole size increases, the curves show that the value of maximum stress decreases and moves towards the intersection. In this study, it was found that the peak stress occurred at the intersection only for crosshole sizes of $c / a=0.25$. The same stress distribution was found for the Mechanica models of these configurations and the von Mises stress and SI distributions through the thickness exhibited similar forms.

\subsection{Definition of the SCF}

The stress concentration effect of the crossholes is quantified in this paper in terms of three elastic SCFs defined in terms of the appropriate maximum calculated stress, and not the stress at the intersection between the crosshole and main bore.

The basic definition of SCF given in equation (1) is redefined in terms of the maximum principal stress $\sigma_{1}$ as

$$
K_{1}=\frac{\sigma_{1 \max }}{\sigma_{\theta \mathrm{c}}}
$$

where $\sigma_{1 \max }$ is the maximum value of $\sigma_{1}$ calculated for the configuration and $\sigma_{\theta \mathrm{c}}$ is the value of maximum principal stress at the inner surface of a similar plain cylinder (the hoop stress) calculated from the Lamé solution. This SCF is appropriate for design calculations related to fracture and fatigue applications.

The von Mises equivalent stress $\sigma_{\mathrm{e}} \mathrm{SCF}$ is appropriate to multi-axial yield load and fatigue calculations 
based on the von Mises criterion and is defined as

$$
K_{\mathrm{e}}=\frac{\sigma_{\mathrm{emax}}}{\sigma_{\mathrm{ec}}}
$$

where $\sigma_{\text {emax }}$ is the maximum value of the von Mises equivalent stress in the vessel and $\sigma_{\mathrm{ec}}$ is the value of equivalent stress at the inner surface of a similar plain cylinder calculated from the Lamé solution.

The Tresca equivalent stress or SI SCF is appropriate to yield load and fatigue calculations for pressure vessel design applications according to the Tresca criterion and is defined as

$$
K_{\mathrm{SI}}=\frac{\mathrm{SI}_{\max }}{\mathrm{SI}_{\mathrm{c}}}
$$

where $\mathrm{SI}_{\max }$ is the maximum value of the Tresca SI in the vessel with a crosshole and $\mathrm{SI}_{\mathrm{c}}$ is the value of SI at the inner surface of a similar plain cylinder from the Lamé solution.

\subsection{Stress concentration factors}

The SCFs calculated in the parametric study are listed in Tables 1, 2, and 3 for $K_{1}, K_{\mathrm{e}}$, and $K_{\mathrm{SI}}$ respectively. Table 1 includes the theoretical value of $K_{\theta}=K_{1}$ for a small hole in a thick cylinder given by equation (2). The FEA values for $K_{1}$ are very close to the theoretical values for the smallest crosshole considered, $c / a=$ 0.01 . However, as the hole size increases, there is notable variation in the tabulated FEA value for a given radius ratio. The results of the study for $K_{1}$, $K_{\mathrm{e}}$, and $K_{\mathrm{SI}}$ are represented graphically as threedimensional surfaces in $(K, b / a, c / a)$ space in Figs 6 , 7 , and 8 respectively. These figures show that the magnitude of SCF varies with both the $b / a$ and $c / a$ ratios. Curves of $K_{1}, K_{\mathrm{e}}$, and $K_{\mathrm{SI}}$ versus $c / a$ ratio are plotted in two dimensions in Figs 9, 10, and 11 respectively, for the range of cylinder ratios $b / a$ considered.

\begin{tabular}{|c|c|c|c|c|c|c|c|c|c|}
\hline \multirow[b]{2}{*}{ Cylinder ratio $b / a$} & \multirow[b]{2}{*}{$K_{1}$, equation (1) } & \multicolumn{8}{|c|}{$K_{1}$ for the following crosshole ratios $c / a$} \\
\hline & & 0.01 & 0.02 & 0.05 & 0.075 & 0.10 & 0.15 & 0.20 & 0.25 \\
\hline 1.40 & 2.99 & 2.96 & 2.92 & 2.82 & 2.78 & 2.76 & 2.77 & 2.86 & 3.01 \\
\hline 1.50 & 3.08 & 3.06 & 3.01 & 2.90 & 2.84 & 2.81 & 2.79 & 2.84 & 2.95 \\
\hline 1.60 & 3.16 & 3.13 & 3.09 & 2.97 & 2.90 & 2.86 & 2.82 & 2.84 & 2.92 \\
\hline 1.75 & 3.26 & 3.24 & 3.19 & 3.06 & 2.98 & 2.93 & 2.87 & 2.87 & 2.91 \\
\hline 2.00 & 3.40 & 3.37 & 3.32 & 3.18 & 3.10 & 3.03 & 2.95 & 2.92 & 2.92 \\
\hline 2.25 & 3.51 & 3.50 & 3.42 & 3.28 & 3.18 & 3.11 & 3.02 & 2.97 & 2.95 \\
\hline 2.50 & 3.59 & 3.58 & 3.50 & 3.35 & 3.25 & 3.18 & 3.07 & 3.01 & 2.98 \\
\hline
\end{tabular}

Table 1 The maximum principal stress SCF $K_{1}$

Table 2 The von Mises equivalent stress SCF $K_{\mathrm{e}}$

\begin{tabular}{lllllllll}
\hline & \multicolumn{7}{c}{$K_{\mathrm{e}}$ for the following crosshole ratios $c / a$} \\
\cline { 2 - 9 } Cylinder ratio b/a & 0.01 & 0.02 & 0.05 & 0.075 & 0.1 & 0.15 & 0.2 & 0.25 \\
\hline 1.4 & 2.77 & 2.74 & 2.67 & 2.64 & 2.63 & 2.66 & 2.76 & 2.91 \\
1.5 & 2.77 & 2.74 & 2.67 & 2.63 & 2.61 & 2.61 & 2.67 & 2.78 \\
1.6 & 2.77 & 2.74 & 2.67 & 2.62 & 2.60 & 2.58 & 2.62 & 2.69 \\
1.75 & 2.78 & 2.74 & 2.66 & 2.62 & 2.58 & 2.55 & 2.56 & 2.61 \\
2 & 2.78 & 2.74 & 2.66 & 2.61 & 2.57 & 2.53 & 2.52 & 2.54 \\
2.25 & 2.78 & 2.75 & 2.66 & 2.61 & 2.57 & 2.52 & 2.50 & 2.50 \\
2.5 & 2.77 & 2.75 & 2.66 & 2.61 & 2.57 & 2.51 & 2.48 & 2.48 \\
\hline
\end{tabular}

Table 3 The SI SCF $K_{\text {SI }}$

\begin{tabular}{lcccccccc}
\hline & \multicolumn{7}{c}{$K_{\mathrm{SI}}$ for the following crosshole ratios $c / a$} \\
\cline { 2 - 8 } Cylinder ratio $b / a$ & 0.01 & 0.02 & 0.05 & 0.075 & 0.10 & 0.15 & 0.20 & 0.25 \\
\hline 1.40 & 2.48 & 2.45 & 2.38 & 2.34 & 2.33 & 2.34 & 2.41 & 2.52 \\
1.50 & 2.49 & 2.45 & 2.37 & 2.33 & 2.30 & 2.30 & 2.33 & 2.41 \\
1.60 & 2.49 & 2.45 & 2.37 & 2.32 & 2.29 & 2.27 & 2.28 & 2.34 \\
1.75 & 2.49 & 2.45 & 2.37 & 2.32 & 2.28 & 2.24 & 2.24 & 2.27 \\
2.00 & 2.49 & 2.45 & 2.36 & 2.31 & 2.27 & 2.22 & 2.20 & 2.20 \\
2.25 & 2.50 & 2.45 & 2.36 & 2.31 & 2.27 & 2.21 & 2.18 & 2.17 \\
2.50 & 2.50 & 2.45 & 2.36 & 2.31 & 2.26 & 2.20 & 2.17 & 2.15 \\
\hline
\end{tabular}




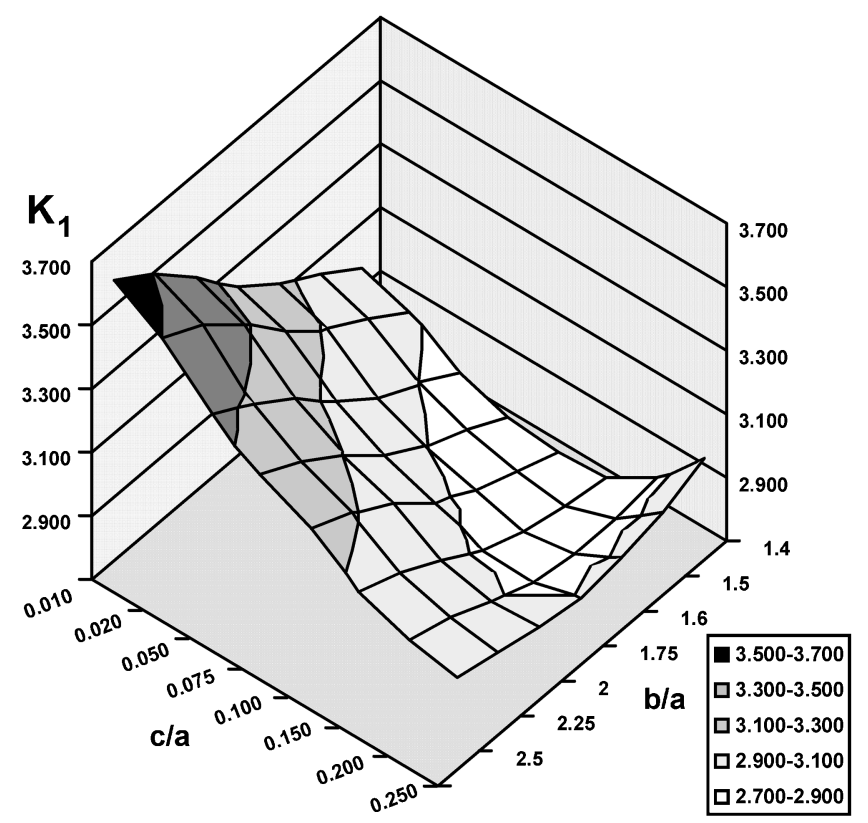

Fig. 6 Variation in $K_{1}$ with $c / a$ and $b / a$

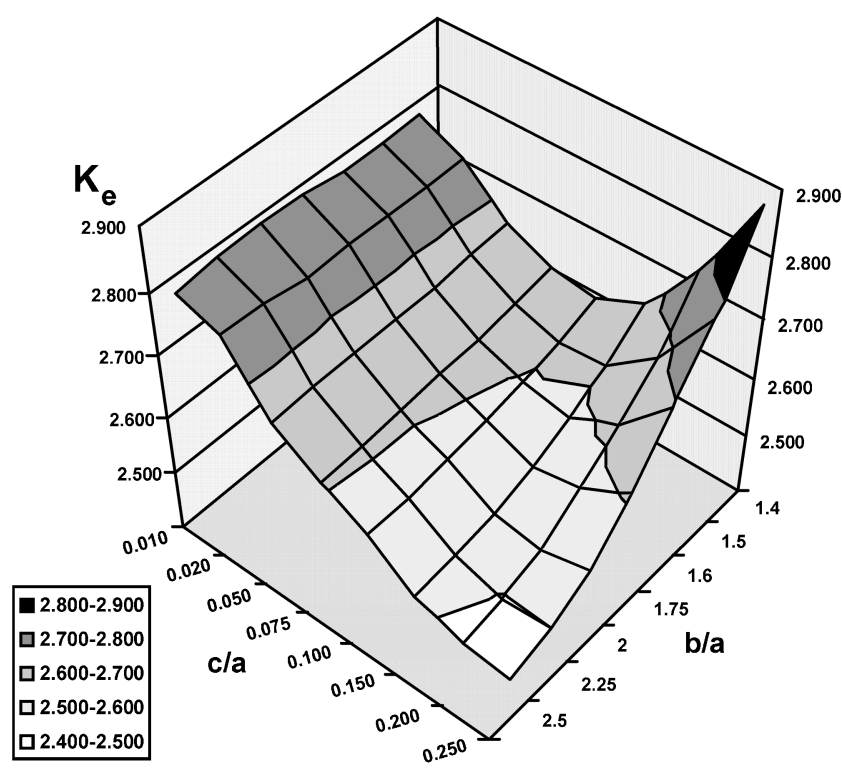

Fig. 7 Variation in $K_{e}$ with $c / a$ and $b / a$

Figure 9 shows that the maximum $K_{1}$ value occurs for the smallest hole, $c / a=0.01$, in the thickest cylinder, $b / a=2.5$. As $c / a$ increases for constant $b / a, K_{1}$ reduces in value over the range considered. A similar behaviour is observed for $b / a=12.25$ and $b / a=2.00$, although in the latter case the curve appears to flatten out between $c / a=0.2$ and $c / a=$ 0.25 . The thinner cylinders show similar curves initially in these cases; $K_{1}$ reduces to a minimum value and then increases for the remainder of the range considered. This behaviour is significantly different from the continuously decreasing $K_{\theta}\left(=K_{1}\right)$

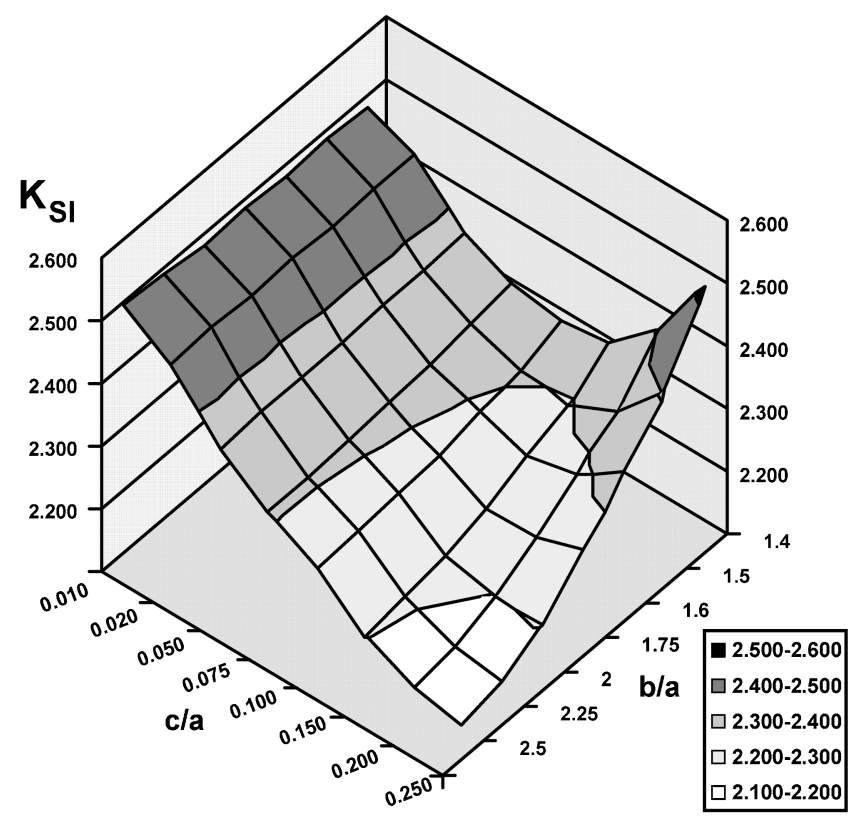

Fig. 8 Variation in $K_{\mathrm{SI}}$ with $c / a$ and $b / a$

curves given by Gerdeen, shown in Fig. 2. The Gerdeen curves for $b / a=1.5,2.0$, and 3.0 for the $c / a$ range considered in the present investigation are shown in Fig. 9; the Gerdeen solution gives higher values of $K_{1}$ than the FEA values.

The crosshole has a lower stress concentration effect on the von Mises equivalent stress than on the maximum principal stress. Table 3 and Fig. 10 show that $K_{\mathrm{e}}$ is essentially independent of cylinder thickness for the smallest hole, $c / a=0.01$, considered. As the crosshole-to-bore-radius ratio increases, increasingly greater differences are found in $K_{\mathrm{e}}$. The highest stress concentration effect in this case is found for the thinnest cylinder considered, $b / a=1.4$, and reduces with increasing thickness. This is the opposite of the trend observed for $K_{1}$. In the case of the thinner cylinders, the SCF initially decreases with increasing $c / a$ to a minimum value and then increases for the remainder of the range. The $K_{\mathrm{SI}}$ curves of Fig. 11 are similar in form to the $K_{\mathrm{e}}$ curves of Fig. 10 but show lower values of SCF.

\section{CONCLUSION}

The results of the parametric FEA show that the maximum stress at a small crosshole in a pressurized cylinder with $v>0$ (in this case, $v=0.3$ ) does not occur at the intersection with the main bore but rather some small distance up the crosshole from the intersection with the main bore. The location of maximum stress concentration moves towards the 


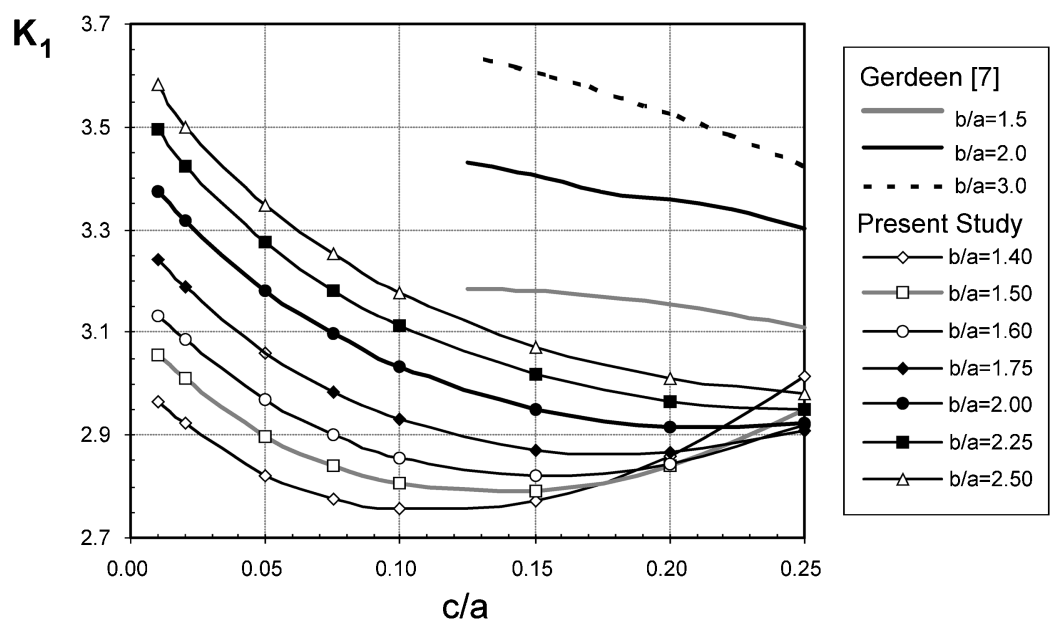

Fig. 9 Variation in $K_{1}$ with the crosshole-to-bore-radius ratio $c / a$

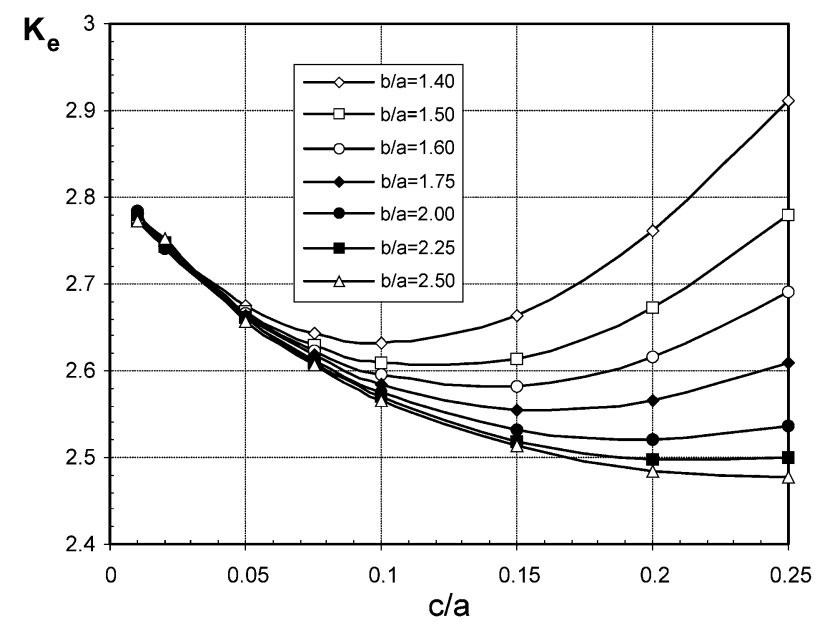

Fig. 10 Variation in $K_{\mathrm{e}}$ with the crosshole-to-boreradius ratio $c / a$

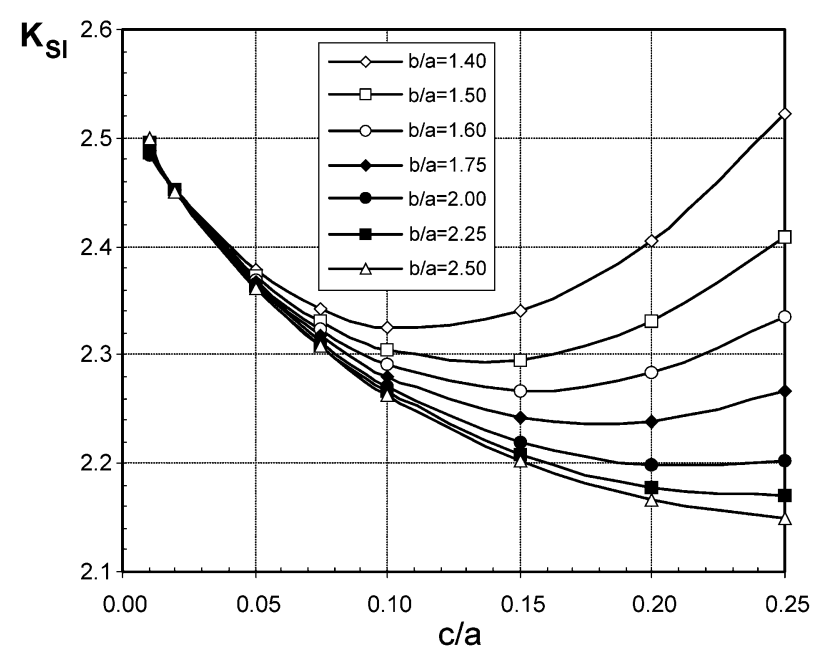

Fig. 11 Variation in $K_{\mathrm{SI}}$ with the crosshole-to-boreradius ratio $c / a$ intersection with increasing crosshole-to-bore-radius ratio and occurs at the intersection for the largest ratio considered in the present investigation, $c / a=$ 0.25 . A practical implication of this is that fatigue life may be enhanced by local polishing inside the crossbore or extending shot peening or cold working up the crossbore, to induce surface compressive residual stresses.

The FEA study showed that for a given cylinder radius ratio $b / a$, the maximum SCF (principal, von Mises, and SI) varied with crosshole-to-main-boreradius ratio $c / a$. The $K_{1} \mathrm{SCF}$ for thicker cylinders exhibited a minimum value within the range considered in the study. In the case of the thinner cylinders, no minima were observed in this range. This finding contradicts the analytical solution obtained by Gerdeen, which predicts a minimum SCF for a cylinder with a sidehole ratio of $c / a=1$. The FEA results presented allow designers to select optimum $b / a$ and $c / a$ ratios to minimize stress concentration in real problems.

\section{ACKNOWLEDGEMENTS}

The use of the ANSYS and Mechanica software under educational licence is acknowledged.

\section{REFERENCES}

1 Love, A. E. H. A treatise on the mathematical theory of elasticity, 4th edition, 1944 (Dover Publications, New York).

2 Timoshenko, S. and Goodier, J. N. Theory of elasticity, 2nd edition, international student edition, 1951 (McGraw-Hill, New York). 
3 Benham, P. P., Crawford, R. J., and Armstrong, C. G. Mechanics of engineering materials, 2nd edition, 1996 (Prentice-Hall, Englewood Cliffs, New Jersey).

4 Fessler, H. and Lewin, B. H. Stress distribution in a tee junction of thick pipes. Br. J. Appl. Physics, 1956, 7, 76-79.

5 Faupel, J. H. and Harris, D. B. Stress concentration in heavy-walled cylindrical pressure vessels. Ind. Engng Chemistry, 1957, 49(12), 1979-1986.

6 Morrison, J. L. M., Crossland, B., and Parry, J. S. C. Fatigue strength of cylinders with cross-bores. J. Mech. Engng Sci., 1959, 1(3), 207-210.

7 Gerdeen, J. C. Analysis of stress concentrations in thick cylinders with sideholes and crossholes. Trans. ASME, J. Engng Industry, 1972, 94, 815-823.

8 Chaaban, A. and Burns, D. J. Design of high pressure vessels with radial crossbores. Physica $B+C, 1986$, 139-140, 766-772.

9 Masu, L. M. and Craggs, G. Fatigue strength of thick-walled cylinders containing cross bores with blending features. J. Mech. Engng Sci., 1992, 206, 299-309.

10 Makulsawatudom, P., Mackenzie, D., and Hamilton, R. Stress concentration at crossholes in thick cylindrical vessels. J. Strain Analysis, 2004, 39(5), 471-481.

11 Gerdeen, J. C. and Smith, R. E. Experimental determination of stress-concentration factors in thick-walled cylinders with crossholes and sideholes. Expl Mechanics, 1972, 12, 503-526.

12 ANSYS version 10.0, 2005 (ANSYS Inc., Canonsburg, Pennsylvania).

13 Pro/Mechanica, 2005 (PTC, Needham, Massachusetts).

14 Sternberg, E. and Sadowsky, M. A. Threedimensional solution for the stress concentration around a circular hole in a plate of arbitrary thickness. J. Appl. Mechanics, 1949, 16, 27-38.

15 Folias, E. S. and Wang, J. J. On the three-dimensional stress field around a circular hole in a plate of arbitrary thickness. Comput. Mechanics, 1990, 6, 379-391.
16 Youngdahl, C. K. and Sternberg, E. Threedimensional stress concentration around a cylindrical hole in a semi-infinite elastic body. J. Appl. Mechanics, 1966, 33, 855-865.

\section{APPENDIX}

\section{Notation}

$a \quad$ inner diameter of the cylinder

$b \quad$ outer diameter of the cylinder

$c \quad$ crosshole radius

$K \quad$ stress concentration factor

$K_{\mathrm{e}} \quad$ von Mises equivalent stress concentration factor

$K_{\mathrm{SI}} \quad$ Tresca stress intensity stress concentration factor

$K_{1} \quad$ maximum principal stress concentration factor

$L \quad$ cylinder half-length

$r \quad$ radial distance from the cylinder axis

SCF stress concentration factor

SI stress intensity of the maximum principal stress difference

$v \quad$ Poisson's ratio

$\sigma_{\mathrm{ec}} \quad$ maximum von Mises equivalent stress of the plain cylinder

$\sigma_{\text {emax }}$ maximum von Mises equivalent stress at the crosshole

$\sigma_{\theta \mathrm{c}} \quad$ maximum hoop or principal stress of the plain cylinder

$\sigma_{\theta \max } \quad$ maximum principal stress at the crosshole $\sigma_{1 \max }$ maximum principal stress of the crosshole cylinder 